\title{
Autophagy in RAW264.7 Cells Treated with Surface-Functionalized Graphene Oxides
}

\author{
Chang Seok Park, ${ }^{1}$ Kyoung Soon Choi, ${ }^{2}$ In Won Park, ${ }^{1}$ Jae Woo Jung, ${ }^{1}$ Jae Chol Choi, ${ }^{1}$ \\ Jae Yeol Kim, ${ }^{1}$ Byoung Whui Choi, ${ }^{1}$ Yu Geun Kim, ${ }^{2}$ Jong Wook Shin, ${ }^{1}$ and Soo Young Kim ${ }^{2}$ \\ ${ }^{1}$ Department of Internal Medicine, Chung-Ang University College of Medicine, 221 Heukseok-dong, Dongjak-gu, \\ Seoul 156-756, Republic of Korea \\ ${ }^{2}$ School of Chemical Engineering and Materials Science, Chung-Ang University, 221 Heukseok-dong, Dongjak-gu, \\ Seoul 156-756, Republic of Korea
}

Correspondence should be addressed to Jong Wook Shin; basthma@cau.ac.kr and Soo Young Kim; sooyoungkim@cau.ac.kr

Received 19 May 2015; Accepted 21 July 2015

Academic Editor: Jin W. Seo

Copyright (C) 2015 Chang Seok Park et al. This is an open access article distributed under the Creative Commons Attribution License, which permits unrestricted use, distribution, and reproduction in any medium, provided the original work is properly cited.

This study investigated cytotoxicity, particularly autophagy, in RAW264.7 cells exposed to graphene oxide (GO) and its derivatives (dodecylamine-GO (DA-GO), reduced GO (rGO), and sodium dodecyl sulfate-rGO (SDS-rGO)). Appearance of amine stretching bands, out-of-plane $\mathrm{C}-\mathrm{H}$ stretching vibrations, and $\mathrm{S}=\mathrm{O}$ stretching bands in infrared spectra indicated the formation of DA-GO, rGO, and SDS-rGO, respectively. Light microscopy and microculture tetrazolium assay showed that all the GO types exerted cytotoxic effects on RAW264.7 cells in a concentration-dependent manner. Higher concentrations of the GO types downregulated the expression of PU.1, a unique transcription factor in monocytes and macrophages, and decreased the conversion of LC3A/BI to LC3A/B-II, suggesting that PU.1 was associated with autophagy in RAW264.7 cells. These results suggested that surfacefunctionalized GOs exerted cytotoxic effects in a concentration-dependent manner by changing the expression of critical genes and inducing autophagy in macrophages.

\section{Introduction}

Nanomaterials have diverse applications because of their extraordinary physicochemical characteristics. In the last decade, several novel nanomaterials have been developed that have found applications in tissue engineering and regenerative medicine. Nanomaterials affect the immune system, especially macrophages and lymphocytes, thus significantly affecting human health [1]. Graphene, which is characterized by a two-dimensional honeycomb lattice carbon structure, is a potential nanomaterial [2]. Different graphene subtypes, for example, graphene made by chemical vapor deposition, graphene nanoribbons, graphene oxide $(\mathrm{GO})$, and reduced GO (rGO), can be obtained by employing different fabrication methods. Uniformly structured graphene is used as an intracellular carrier in immune cells [3]. Its favorable biological properties such as interaction with RNA and DNA, cellular adhesion, cellular uptake, antibacterial activity, and good biodegradability have increased its application in the biological field. This in turn will increase the industrial production of graphene. As a result, graphene dust may inevitably spread into the environment and may exert harmful effects on human health. Therefore, it is important to analyze the nanosafety and nanotoxicity of graphene materials.

Unique properties of graphene are important under in vivo or intracellular conditions as well as under ex vivo or extracellular conditions. GO and rGO effectively inhibit the growth of bacteria, with minimal cytotoxic effects [4]. RAW264.7 mouse macrophage cell line has long been used as an in vitro model for studying the response of inflammatory molecules to various synthetic stimuli. Macrophages are the first immune response cells that represent the innate immune system. Macrophage response is critical for survival at cell, tissue, organ, and system levels. Therefore, dose- and sizerelated effects of GO or rGO on the morphology, viability, 
and mortality of RAW264.7 cells should be considered while developing biomedical applications of GO. Most previous studies have used rGO or GO without any surface treatments. However, the physicochemical properties of GO depend on its surface [5]. The most critical issue associated with the use of rGO or GO in biomedical applications is to establish versatile functionalization methods for producing GOs that are surface functionalized with biomolecules or biomaterials, with minimal detrimental effects on the bioactivity of these compounds. Therefore, the effect of surface-functionalized GOs on the viability of RAW264.7 cells should be analyzed to determine their nanosafety. Furthermore, surface-functionalized GO-induced intracellular molecular and genetic mechanisms in RAW264.7 cells must be elucidated for determining health care and environmental controls.

Cell death occurs in the form of necrosis, apoptosis, and other forms of programmed or nonprogrammed cell death, including autophagy, which has been acknowledged recently. Autophagy is defined as nanomaterial-associated cellular injury [6]. Autophagy involves degradation of intracellular components in response to stress, and it is negatively controlled by mammalian target of rapamycin complex 1 (mTORC1). Inhibition of mTORC1 kinase activity promotes the formation of an autophagosome containing a complex composed of Beclin 1 and other factors. Formation of the autophagosome also involves the conversion of microtubuleassociated protein light chain 3 (LC3A/B-I) to its lipidated form LC3A/B-II. Thus, conversion of LC3A/B-I to LC3A/B-II is a common indicator of autophagy. A study on mechanisms underlying the death of RAW264.7 cells is essential to elucidate the effects of surface-functionalized GOs on the viability of these cells. Therefore, the present study investigated the differential cellular effects of surface-functionalized GOs on the viability of and gene expression in monocytes and macrophages.

GO was produced using a modified Hummers method, and rGO was produced using hydrazine hydrate [7]. Dodecylamine (DA) and sodium dodecyl sulfate (SDS) were used to functionalize GO or rGO. Fourier transform infrared spectroscopy (FTIR) was used to determine the results of surface functionalization. Optical microscopy was used to investigate cell viability. The measurements obtained were used to elucidate the effects of GO, DA-GO, rGO, and SDSrGO on the viability of RAW264.7 cells and the mechanisms underlying their death.

\section{Materials and Methods}

2.1. Synthesis of GO. GO was prepared using a modified Hummers method. Briefly, $2 \mathrm{~g}$ graphite powder (universal grade, 200 mesh, 99.9995\%; Alfa Aesar) was stirred with $2 \mathrm{~g}$ $\mathrm{NaNO}_{3}$ and $100 \mathrm{~mL}$ concentrated $\mathrm{H}_{2} \mathrm{SO}_{4}$ for 1 day in an ice water bath. After stirring, $12 \mathrm{~g} \mathrm{KMnO}_{4}$ was gradually added to the solution. After thoroughly mixing the solution, the ice bath was removed and the solution was stirred at $35^{\circ} \mathrm{C}$ until a highly viscous liquid was obtained. Next, $200 \mathrm{~mL}$ pure water and $\mathrm{H}_{2} \mathrm{O}_{2}$ were added sequentially to the viscous liquid, and the mixture was centrifuged at $8000 \mathrm{rpm}$ and washed with
$\mathrm{HCl}$ and water. The centrifugation and washing steps were repeated at least five times. Finally, the GO obtained was dried at $50^{\circ} \mathrm{C}$ for $24 \mathrm{~h}$ in a vacuum oven.

2.2. Synthesis of DA-GO. GO was dispersed in $200 \mathrm{~mL}$ deionized water to obtain a concentration of $2 \mathrm{mg} / \mathrm{mL}$. The dispersed GO was ultrasonicated for $30 \mathrm{~min}$ by using WUC$\mathrm{A} 03 \mathrm{H}$ bath-type sonicator (Daihan Scientific, Korea). The GO dispersion was then centrifuged for $15 \mathrm{~min}$ at $3000 \mathrm{rpm}$ to remove unexfoliated GO. Next, $100 \mathrm{~mL}$ of the browncolored dispersion was transferred to a $250 \mathrm{~mL}$ beaker. DA $(0.1853 \mathrm{~g})$ dissolved in $100 \mathrm{~mL}$ ethanol was added to the GO dispersion, and the solution was stirred at room temperature for $2 \mathrm{~h}$. Nucleophilic substitution occurred between the amino moiety of DA and the epoxy moiety of GO. This DAfunctionalized GO dispersion was washed with ethanol to remove excess DA adsorbed on the surface of the modified GO. The reaction product was washed with deionized water to remove excess ethanol. The dark brown powder was dried under vacuum at $60^{\circ} \mathrm{C}$ for $24 \mathrm{~h}$ to obtain DA-GO.

2.3. Synthesis of $r G O$. rGO was synthesized from GO by using hydrazine hydrate. First, $0.1 \mathrm{~g}$ GO was dispersed in $100 \mathrm{~mL}$ deionized water by ultrasonication. Next, $1 \mathrm{~mL}$ hydrazine hydrate $\left(\mathrm{N}_{2} \mathrm{H}_{4}\right.$ [50\%-60\%], reagent grade; Sigma-Aldrich) was added to the GO dispersion. After stirring for $10 \mathrm{~min}$, the solution was transferred to an oil bath fitted with a watercooled condenser, and it was heated at $90^{\circ} \mathrm{C}$ for $24 \mathrm{~h}$. The reaction product was washed with deionized water to remove excess hydrazine hydrate. The final product was then dried in a vacuum oven at $60^{\circ} \mathrm{C}$.

2.4. Synthesis of SDS-rGO. For SDS modification, rGO was dispersed in $200 \mathrm{~mL}$ deionized water to achieve a concentration of $2 \mathrm{mg} / \mathrm{mL}$ by performing ultrasonication for 30 min in VCX-750 probe-type sonicator (Sonics \& Materials, Inc., USA). Next, 14.419 g SDS (ACS regent, $\geq 99.0 \%$; SigmaAldrich) was added to the rGO dispersion, and the solution was stirred at room temperature for $2 \mathrm{~h}$. The SDS-modified rGO (SDS-rGO) dispersion was then washed with deionized water to remove excess SDS. The black powder obtained was dried under vacuum at $60^{\circ} \mathrm{C}$ for $24 \mathrm{~h}$.

2.5. Materials. Dulbecco's modified Eagle's medium (DMEM; Gibco), fetal bovine serum (FBS; Gibco), penicillin/ streptomycin (Gibco), 3-(4,5-dimethylthiazol-2-yl)-2,5diphenyltetrazolium bromide (MTT), NaCl, Tween-20, Trizma base, glycine, and $\mathrm{HCl}$ were purchased from SigmaAldrich. PRO-PREP Protein Extraction Kit and SMART BCA Protein Assay Kit were purchased from Intron. Antibodies against glyceraldehyde-3-phosphate dehydrogenase (GAPDH), pan-actin (p-Act), LC3A/B-I, LC3A/B-II, and PU.1 were purchased from Cell Signaling.

2.6. Cell Culture and Treatment with the GO Types. Murine RAW264.7 macrophages were purchased from ATCC and cultured in DMEM supplemented with $10 \%$ FBS, $100 \mu \mathrm{g} / \mathrm{mL}$ streptomycin, and $100 \mathrm{U} / \mathrm{mL}$ penicillin at $37^{\circ} \mathrm{C}$ in $5 \% \mathrm{CO}_{2}$ atmosphere. Four GO types, that is, GO, DA-GO, rGO, and 
SDS-rGO, were used in the study. For treatment with each GO type, the cells were seeded in proper culture plates (24well plates, $\sim 100 \varphi$, and $100 \mathrm{~mm}$ diameter) at an appropriate seeding density and cultured overnight with indicated concentrations of each GO type for 24 or $48 \mathrm{~h}$.

2.7. Light Microscopy. Cell morphology was examined under a phase-contrast microscope (Olympus IX71) at 100x magnification. RAW264.7 cells were plated in 12-well plates (cell density, $2.5 \times 10^{5}$ cells per well) and were incubated for $24 \mathrm{~h}$ for stabilization. The cells were then treated with GO, DA$\mathrm{GO}, \mathrm{rGO}$, and SDS-rGO at indicated concentrations $(5,25$, and $50 \mu \mathrm{g} / \mathrm{mL}$ for DA-GO and 5, 50, and $200 \mu \mathrm{g} / \mathrm{mL}$ for GO, rGO, and SDS-rGO, resp.) for 24 or $48 \mathrm{~h}$. RAW264.7 cells cultured in a complete medium without any GO type were used as controls. All images were recorded and digitalized using Olympus DP70 and its software program.

2.8. Cytotoxicity Assay. Cytotoxicity of each GO type was evaluated by performing MTT assay. Briefly, $5 \mathrm{mg} / \mathrm{mL}$ MTT solution was prepared in phosphate buffered saline (PBS; Gibco). The solution was sterilized using a syringe filter $(0.2 \mu \mathrm{m}$ pore size, GVS). RAW264.7 cells were plated in 24-well plates (cell density, $1.5 \times 10^{5}$ cells per well) and were incubated for $24 \mathrm{~h}$ for stabilization. The cells were then treated separately with GO, DA-GO, rGO, and SDS-rGO at concentrations ranging from 800 to $0.8 \mu \mathrm{g} / \mathrm{mL}$, which were obtained by diluting the GO types with the culture medium. RAW264.7 cells cultured in a medium without the GO types were used as controls. Culture medium without the RAW264.7 cells or GO types was used as blank. After 24 and $48 \mathrm{~h}$ of incubation, the cells were rinsed twice with cold sterile PBS. Next, 10\% MTT solution in the complete medium was added to each well, and the cells were incubated for additional $4 \mathrm{~h}$ at $37^{\circ} \mathrm{C}$. Next, $100 \mu \mathrm{L} 10 \%$ SDS solution was added to each well, and the cells were incubated for more than $4 \mathrm{~h}$ at $37^{\circ} \mathrm{C}$ to dissolve formazan precipitates in viable cells. Liquefied samples were agitated for $30 \mathrm{~s}$ to mix cells with GO thoroughly. Next, the samples were centrifuged at $14000 \mathrm{rpm}$ for $30 \mathrm{~min}$ to precipitate the GO derivatives. Optical density was measured at $540 \mathrm{~nm}$ by using a microplate reader (Spectramax 340PC384; Molecular Devices), with $670 \mathrm{~nm}$ as the reference wavelength. The experiment was repeated thrice.

2.9. Protein Extraction and Western Blot Analysis. Each GO type was categorized into 3 groups based on its concentration (low, medium, and high concentrations). The concentrations were 5,25 , and $50 \mu \mathrm{g} / \mathrm{mL}$ for DA-GO and 5, 50, and $200 \mu \mathrm{g} / \mathrm{mL}$ for the other GO types. Total proteins were extracted by rinsing the treated cells twice with ice-cold PBS and subsequently lysing the cells in the PRO-PREP solution by using cell scrappers. The extracted components were incubated at $-20^{\circ} \mathrm{C}$ for $20 \mathrm{~min}$ and then centrifuged at $15000 \mathrm{rpm}$ and $4^{\circ} \mathrm{C}$ for $30 \mathrm{~min}$. Clear supernatants containing total proteins were collected, and protein concentrations were determined using the SMART BCA Protein Assay Kit. Equal amounts of proteins were separated by performing sodium dodecyl sulfate-polyacrylamide gel electrophoresis and were transferred onto polyvinylidene fluoride membranes. The membranes were blocked with $5 \%$ nonfat milk and were incubated overnight with primary antibodies at $4^{\circ} \mathrm{C}$ with gentle agitation. The membranes were then incubated with appropriate secondary antibodies, and protein bands were detected using Dogen Pico or Femto Chemiluminescence Kit. Densities of protein bands were quantified using Image Lab imaging processing program. Because the extent of the conversion of LC3A/B-I to LC3A/B-II is correlated with the level of autophagy, levels of LC3A/B-I and LC3A/B-II were detected by performing western blot analysis. Conversion of $\mathrm{LC} 3 \mathrm{~A} / \mathrm{B}$ was determined using the following formula: band density of LC3A/B-II/(band density of LC3A/B-I + band density of LC3A/B-II). Expression of p-Act, GAPDH (internal control), and PU.1 (transcription factor that serves an indicator of RAW264.7 cells) was also examined.

\section{Results and Discussion}

Figure 1 shows the FTIR spectra of (a) GO, (b) DA-GO, (c) rGO, and (d) SDS-rGO. Expected structures of each GO type are shown in the inset of Figure 1. A broad peak at $3437 \mathrm{~cm}^{-1}$ corresponding to $\mathrm{O}-\mathrm{H}$ stretching was observed for all the samples, suggesting that $\mathrm{rGO}$ and SDS-rGO contained some oxygen bonds even though their oxygen content decreased after reduction. Another peak at $1636 \mathrm{~cm}^{-1}$ was also observed for all the samples, indicating the presence of graphitic $\mathrm{sp}_{2}$ bonds. Peaks around $1700-1733 \mathrm{~cm}^{-1}$ and at $1272 \mathrm{~cm}^{-1}$ were observed for GO, indicating the presence of carboxyl and epoxy groups, respectively. However, these peaks were not observed for other samples, suggesting that the carboxyl and/or epoxy groups reacted with DA in DA-GO and were reduced in $\mathrm{rGO}$ and SDS-rGO. The remaining peaks for GO were observed at $1380\left(\mathrm{CH}_{3}\right.$ bending $)$ and $1056 \mathrm{~cm}^{-1}$, which is typical for primary alcohols [8]. New peaks at 2955, 2922, and $2852 \mathrm{~cm}^{-1}$ for DA-GO corresponded to C-H stretching vibrations of $\mathrm{CH}_{3}, \mathrm{CH}_{2}$, and, $\mathrm{CH}$ groups, respectively. Peaks at $1644,1457,1265$, and $1012 \mathrm{~cm}^{-1}$ for DA-GO corresponded to carbonyl stretching vibrations of an amide-carbonyl bond, an amine group, an amine stretching band, and a primary alcohol in phenolic compounds, respectively $[9,10]$. Presence of these peaks confirmed that GO was successfully modified with DA. Peaks at 998 and $990 \mathrm{~cm}^{-1}$ for rGO and SDS-rGO, respectively, corresponded to out-of-plane $\mathrm{C}-\mathrm{H}$ stretching vibrations and suggested sufficient reduction of GO. A new peak at $1168 \mathrm{~cm}^{-1}$ for SDS-rGO corresponded to $S=O$ stretching and indicated that small amounts of SDS existed on the surface of rGO. These data indicated that all the samples were modified as intended.

The morphology of a cell is an important indicator of its viability. Figure 2 shows microscopic images of RAW264.7 cells treated with (a) GO, (b) DA-GO, (c) rGO, and (d) SDS-rGO. The cells were exposed to 3 concentrations of GOs. Researchers or workers are likely to be exposed to low concentrations $(5 \mu \mathrm{g} / \mathrm{mL})$ of GOs. Intermediate $(50 \mu \mathrm{g} / \mathrm{mL})$ and high $(200 \mu \mathrm{g} / \mathrm{mL})$ concentrations of GO were examined to determine the effect of long-term exposure to GOs. Cellular morphology was conserved in almost all the cells 


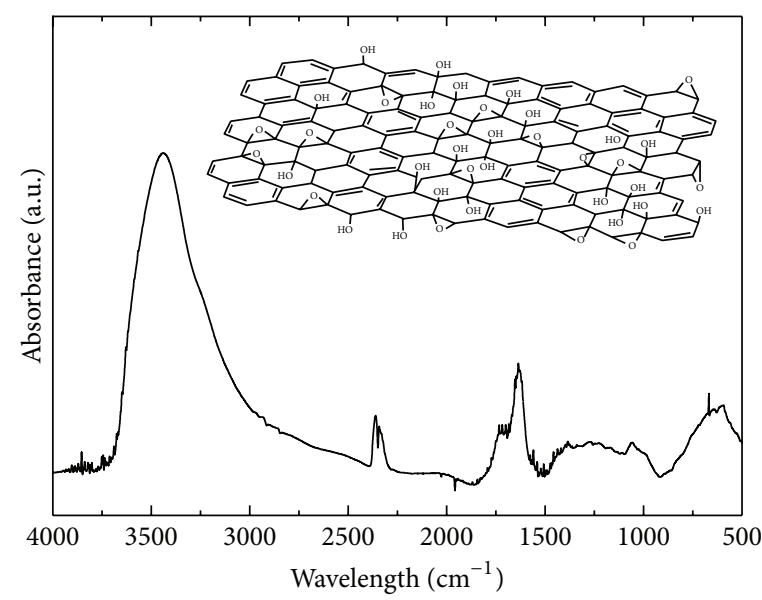

(a) GO

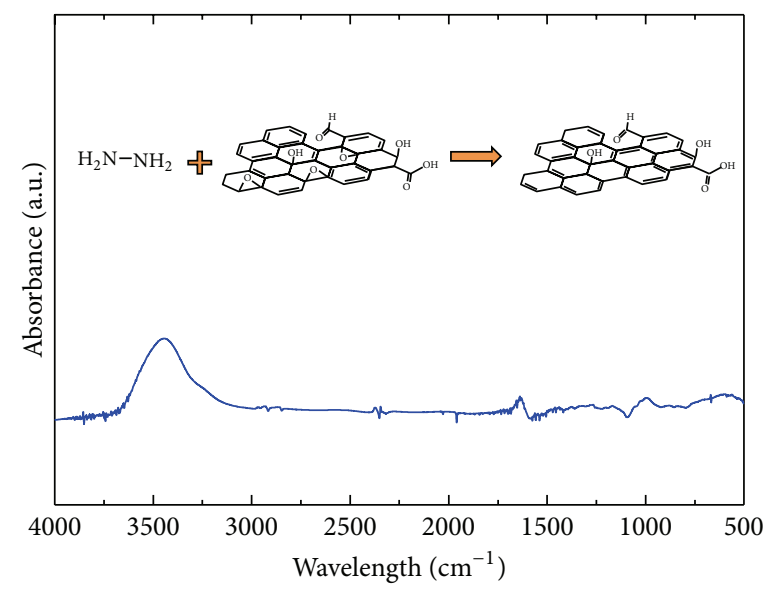

(c) rGO

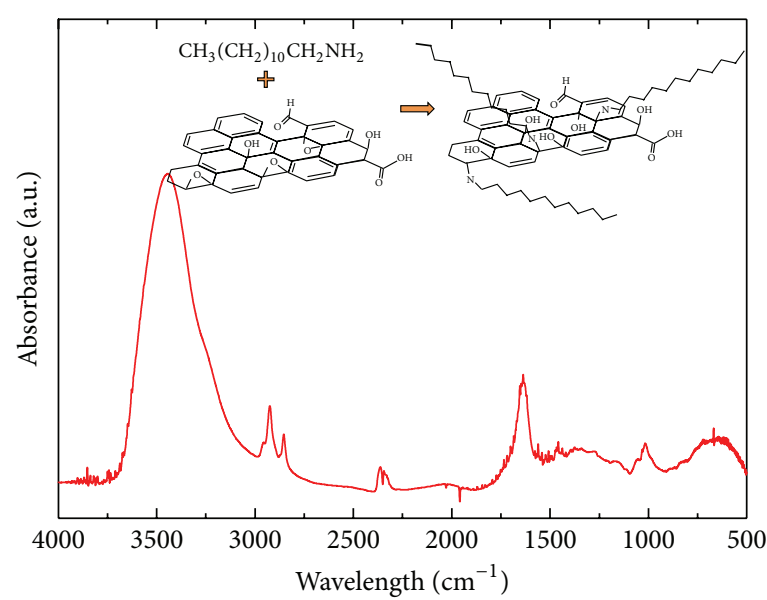

(b) DA-GO

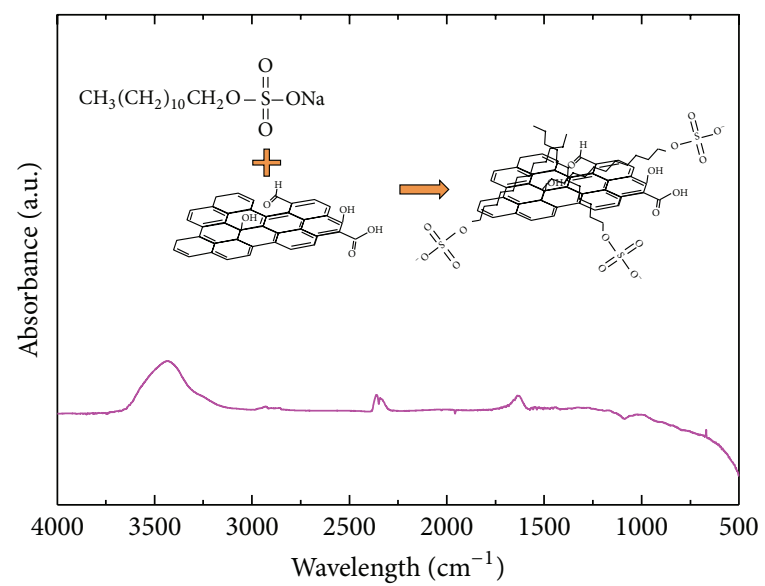

(d) SDS-rGO

FIGURE 1: FTIR spectra of (a) GO, (b) DA-GO, (c) rGO, and (d) SDS-rGO.

exposed to lower concentrations of each GO type. However, cells exposed to higher concentrations of all the GO types had dysmorphic appearance. RAW264.7 cells showed loosely adherent, spindle, ovoid, or round shapes, which are characteristics of injured cells. Furthermore, cellular debris was detected in cell samples treated with all the GO types, which increased with an increase in the concentration of the GO types.

Figure 3 shows the cytotoxicity of various GO types determined by performing the MTT assay. Cell viability decreased with an increase in the concentration of GOs, regardless of the GO type. Cell viability decreased linearly in cells treated with GO and decreased rapidly in cells treated with higher concentrations $(>25 \mu \mathrm{g} / \mathrm{mL}$ ) of $\mathrm{rGO}$ and SDS-rGO. Cells treated with $0.8 \mu \mathrm{g} / \mathrm{mL}$ DA-GO showed remarkably decreased viability that was also observed in cells treated with $12.5 \mu \mathrm{g} / \mathrm{mL}$ DA-GO. Cells treated with DA-GO concentrations higher than $12.5 \mu \mathrm{g} / \mathrm{mL}$ showed a rapid decrease in viability, suggesting that GO types with different functional groups exerted different cytotoxic effects. No difference was observed in the concentration-dependent decrease in cell viability between cells treated for 24 and $48 \mathrm{~h}$.
Figure 4 shows the results of western blot analysis of cells treated with (a) GO, (b) DA-GO, (c) rGO, and (d) SDS-rGO. Expression of p-Act decreased in a dose-dependent manner in all the samples. In contrast, expression of GAPDH was constant in all the samples. Therefore, GAPDH was used as a housekeeping gene for normalizing the semiquantitative analysis of gene expression. PU.1, NF $\kappa$ B, and AP-1 are representative transcription factors in cells belonging to the monocyte lineage. Surface-functionalized GOs downregulated the expression of PU.1 in a concentration-dependent manner. The ratio of conversion of LC3A/B-I to LC3A/B-II also decreased in a dose-dependent manner except for RAW264.7 cells with SDS-rGO at a concentration of $200 \mu \mathrm{g} / \mathrm{mL}$ for $48 \mathrm{~h}$, which was similar to that observed with PU.1.

Based on these observations, the effects of GO and its functionalized derivatives on autophagy in monocytes/ macrophages can be explained as follows. Macrophages engulf graphene materials and convert them into biodegradable intracellular nanomaterials $[11,12]$. Macrophages inhibit the endocytosis of polyethylene glycol- and bovine serum albumin-functionalized graphene but stimulate the endocytosis of polyetherimide-functionalized graphene $[13,14]$. 


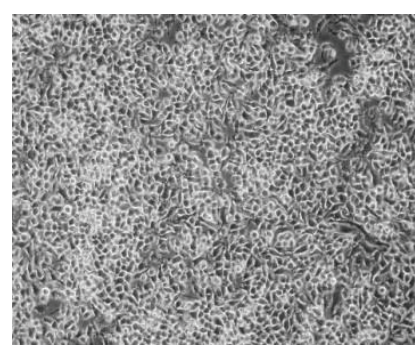

Control

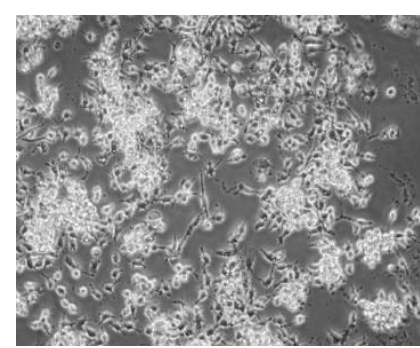

$5 \mu \mathrm{g} / \mathrm{mL}$

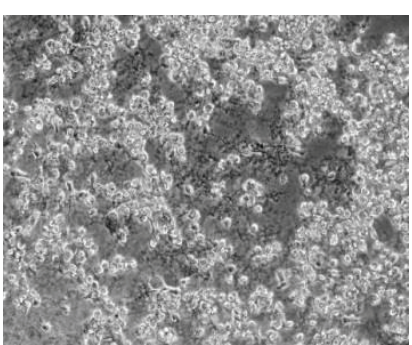

$50 \mu \mathrm{g} / \mathrm{mL}$

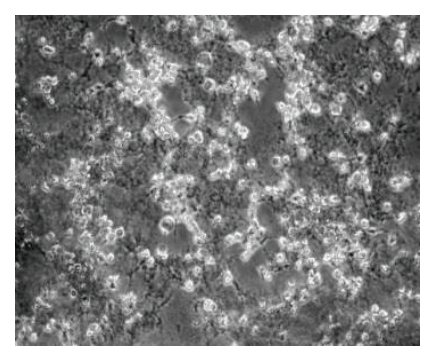

$200 \mu \mathrm{g} / \mathrm{mL}$

(a)

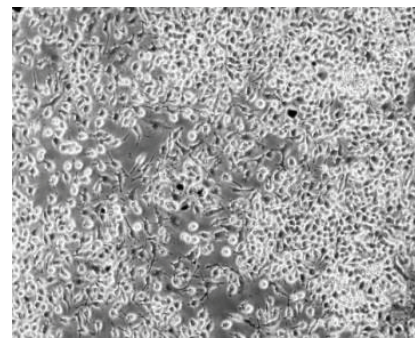

$5 \mu \mathrm{g} / \mathrm{mL}$

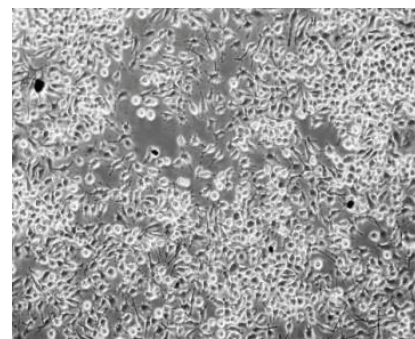

$5 \mu \mathrm{g} / \mathrm{mL}$

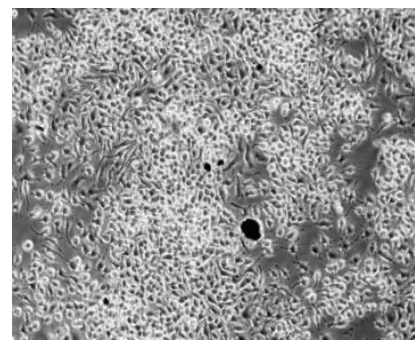

$5 \mu \mathrm{g} / \mathrm{mL}$

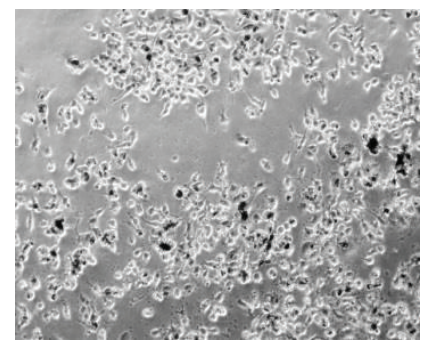

$25 \mu \mathrm{g} / \mathrm{mL}$

(b)

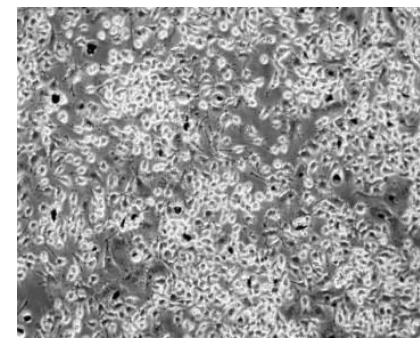

$50 \mu \mathrm{g} / \mathrm{mL}$

(c)

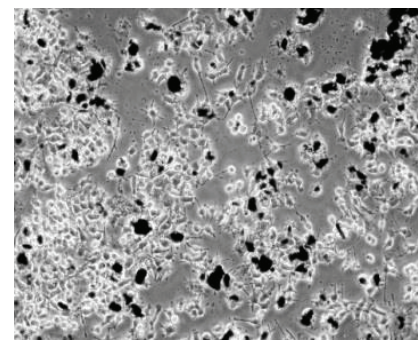

$50 \mu \mathrm{g} / \mathrm{mL}$

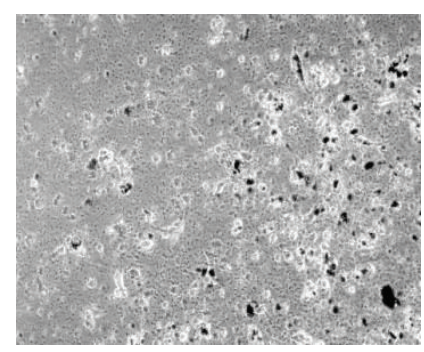

$50 \mu \mathrm{g} / \mathrm{mL}$

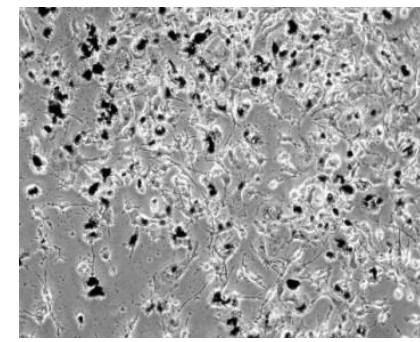

$200 \mu \mathrm{g} / \mathrm{mL}$

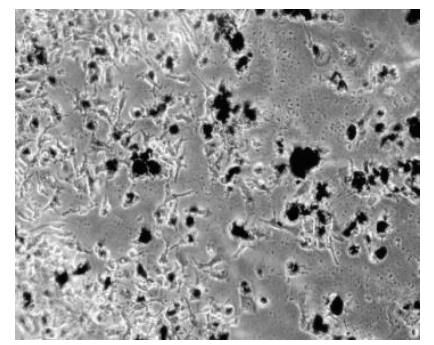

$200 \mu \mathrm{g} / \mathrm{mL}$

(d)

FIGURE 2: Microscopic images showing morphological changes in RAW264.7 cells exposed to (a) GO, (b) DA-GO, (c) rGO, and (d) SDS-rGO at the indicated concentrations for $24 \mathrm{~h}$. Exposure to each GO type exerted cytotoxic effects on RAW264.7 cells.

These results indicate that surface-functionalized graphene exerts diverse effects from biodegradation to nanotoxicity that may injure various organs [15].

Graphene induces apoptosis, autophagy, and inflammation in macrophages through $\mathrm{NF} \kappa \mathrm{B}$ or p38 MAPK by increasing the expression of TNF- $\alpha[16,17]$. Autophagy is a type of programmed cell death, like apoptosis. Autophagy involves multiple processes such as phagocytosis or endocytosis, formation of the autophagosome, recycling of intracellular proteins, and degradation of extracellular antigens. At the cellular level, autophagy can determine cell fate, that is, survival or death. LC3 processing is a marker of autophagy because it is a major step in the formation of the autophagosome. Generally, conversion of LC3A/B-I to LC3A/B-II indicates the activation of autophagy because it involves ubiquitinaselike enzymes that cause the selective degradation of target molecules [18]. In the present study, the ratio of conversion of LC3A/B-I to LC3A/B-II decreased in a dose-dependent 


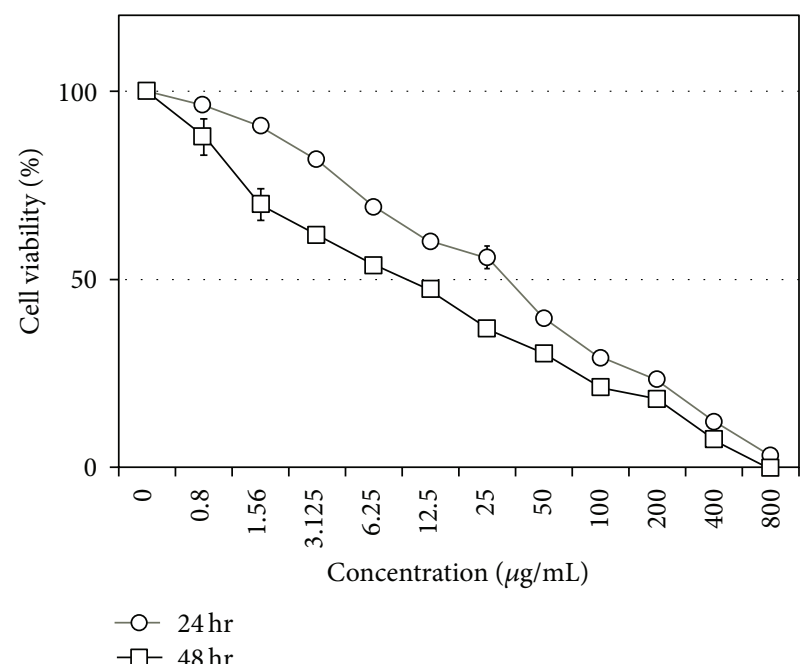

(a) GO

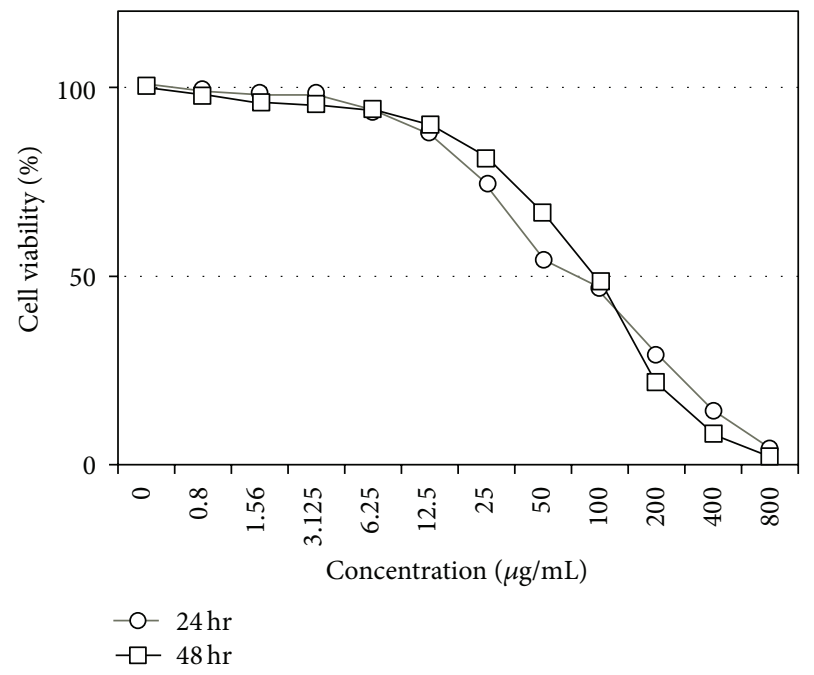

(c) rGO

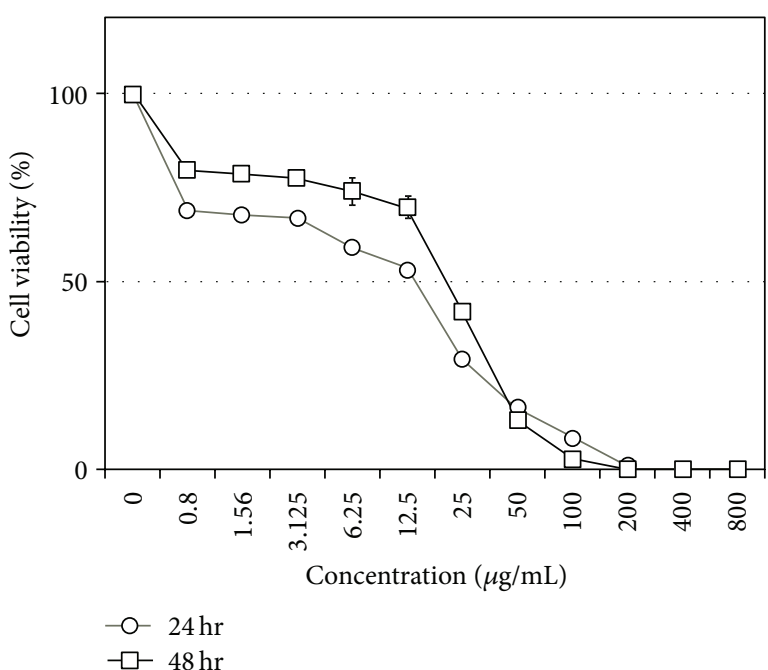

(b) DA-GO

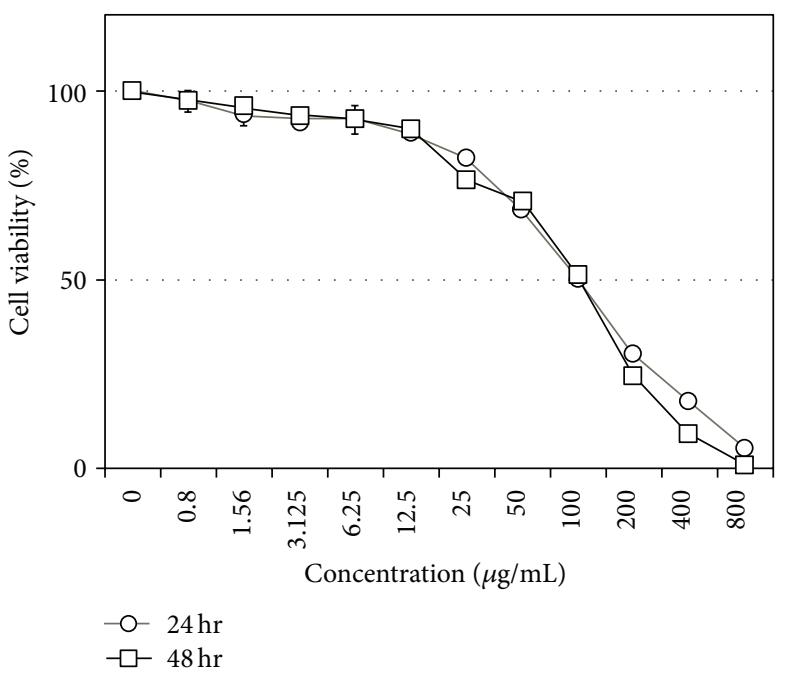

(d) SDS-rGO

FIGURE 3: Viability of RAW264.7 cells treated with (a) GO, (b) DA-GO, (c) rGO, and (d) SDS-rGO.

manner (Figure 4), suggesting that autophagy was one of the cell death processes in macrophages with the different degrees of contribution. PU.1 is one of the transcription factors in monocytes and plays pivotal roles in the expression of various genes $[19,20]$. Expression of PU.1 indicates the involvement of apoptotic pathways [21, 22]. Interestingly, exposure of RAW246.7 cells to higher concentrations of GO types decreased the expression of PU.1 and conversion of LC3A/B-I to LC3A/B-II. These results suggest that PU.1 plays a regulatory role in inducing autophagy in RAW246.7 cells. Furthermore, GAPDH expression was not affected by the increase in the concentrations of GO types. To the best of our knowledge, this is the first report on constant GAPDH expression in macrophages exposed to surfacefunctionalized GOs. Therefore, GAPDH may be used instead of $\mathrm{p}$-Act to normalize gene expression in macrophages. These results highlight the need for further studies to determine mechanisms underlying immune response or cellular toxicity, including autophagy, to establish strategies for preventing or treating respiratory and immunological toxicities in humans. Furthermore, these results indicate that appropriate preparation of GOs is necessary in manufacturing plants and industrial environments.

\section{Conclusion}

This study investigated the cytotoxic effects of GO, DA-GO, rGO, and SDS-rGO on RAW264.7 cells. Appearance of amine stretching bands, out-of-plane $\mathrm{C}-\mathrm{H}$ stretching vibrations, and $\mathrm{S}=\mathrm{O}$ stretching in FTIR spectra indicated the formation of DA-GO, rGO, and SDS-rGO, respectively. Light microscopy and MTT assay showed that all the GO types exerted cytotoxic effects in a concentration-dependent manner. Western blot analysis confirmed cytotoxicity, decreased expression 

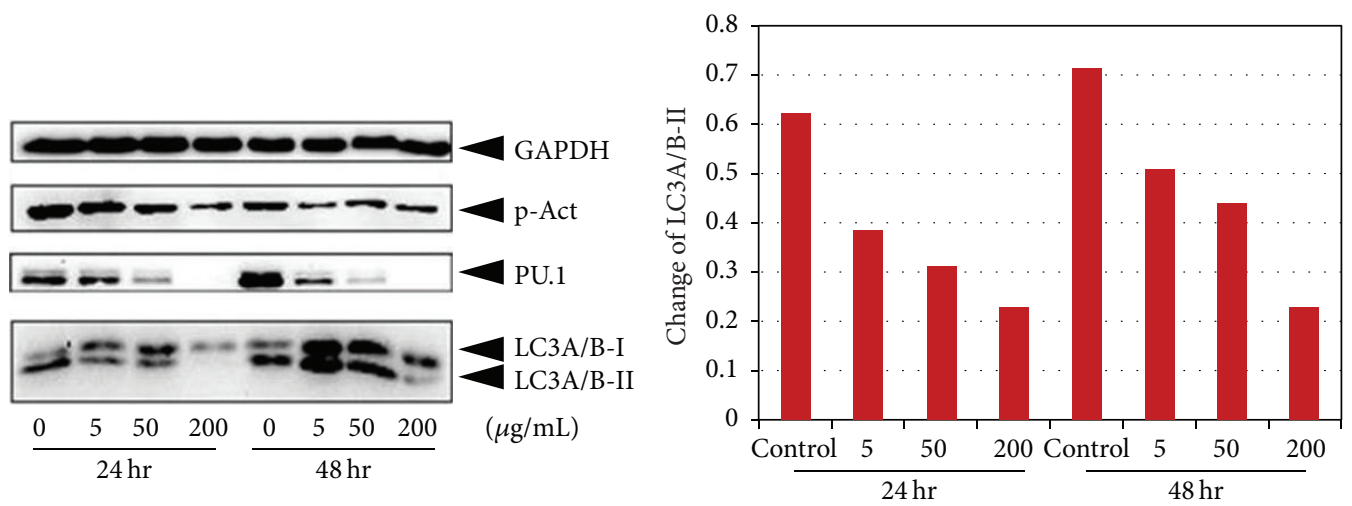

(a) GO
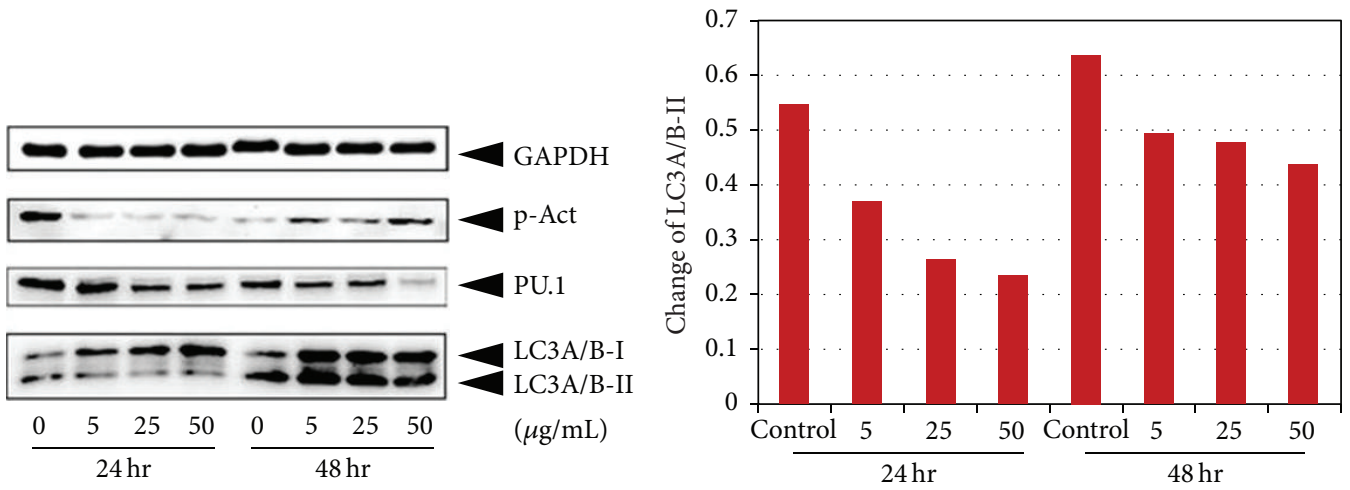

(b) DA-GO
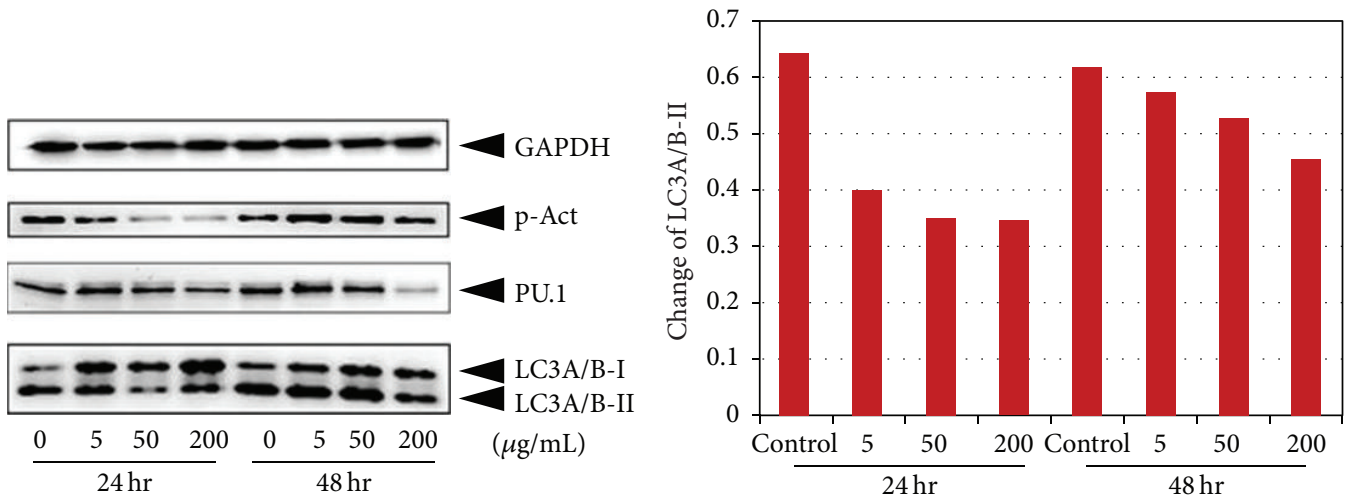

(c) $\mathrm{rGO}$
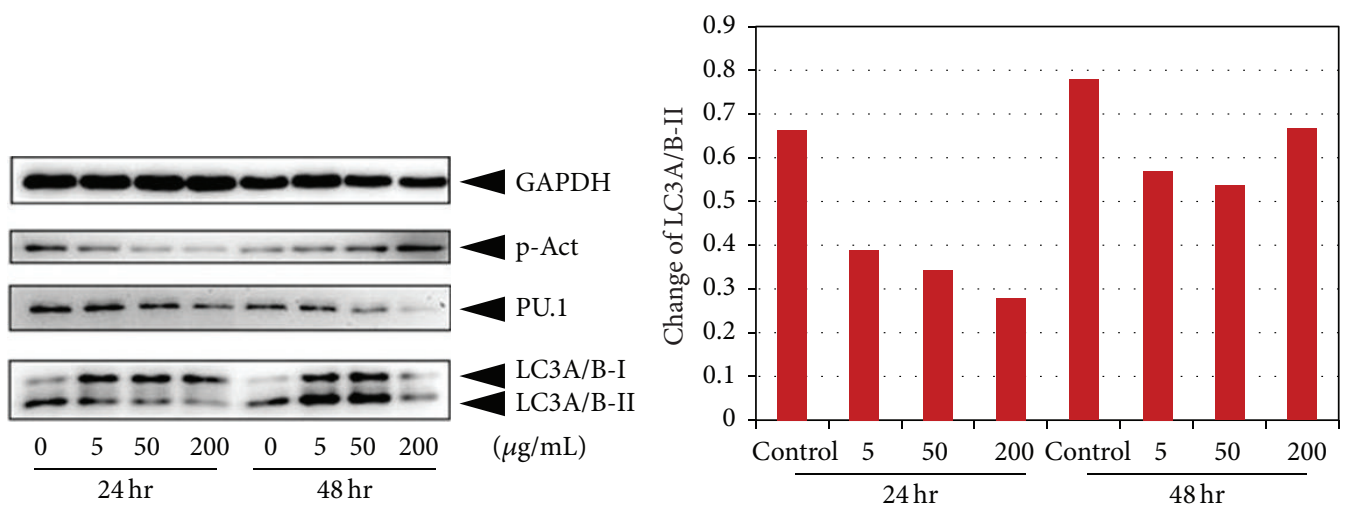

(d) SDS-rGO

FIGURE 4: Expression of GAPDH, p-Act, PU.1, and autophagy marker proteins in RAW264.7 cells treated with the different concentrations of GO types for different treatment durations. (a) Representative results of western blot analysis of each protein. (b) Changes in the ratio of $\mathrm{LC} 3 \mathrm{~A} / \mathrm{B}-\mathrm{II} /(\mathrm{LC} 3 \mathrm{~A} / \mathrm{B}-\mathrm{I}+\mathrm{LC} 3 \mathrm{~A} / \mathrm{B}-\mathrm{II})$ as a function of GO concentration and treatment duration. Level of LC3A/B-I and LC3A/B-II expression was analyzed by western blot analysis by using specific antibodies. 
of PU.1, and induction of autophagy in a concentrationdependent manner in macrophages treated with the $4 \mathrm{GO}$ types. These results suggested that surface-functionalized GOs exerted cytotoxic effects in a concentration-dependent manner by changing the expression of critical genes and by inducing autophagy.

\section{Conflict of Interests}

The authors declare that there is no conflict of interests regarding the publication of this paper.

\section{Authors' Contribution}

Chang Seok Park and Kyoung Soon Choi contributed equally to this work.

\section{Acknowledgments}

This work was supported in part by a Research Scholarship Grant of 2015 from the Chung-Ang University and by a grant from the National Research Foundation of Korea that is funded by the Korean government (MSIP; no. 2014R1A2A1A11051).

\section{References}

[1] M. Orecchioni, D. Bedognetti, F. Sgarrella, F. M. Marincola, A. Bianco, and L. G. Delogu, "Impact of carbon nanotubes and graphene on immune cells," Journal of Translational Medicine, vol. 12, no. 1, article no. 138, 2014.

[2] K. C. Kwon, K. S. Choi, B. J. Kim, J.-L. Lee, and S. Y. Kim, "Work-function decrease of graphene sheet using alkali metal carbonates," The Journal of Physical Chemistry C, vol. 116, no. 50, pp. 26586-26591, 2012.

[3] J. Sun, J. Chao, J. Huang et al., "Uniform small graphene oxide as an efficient cellular nanocarrier for immunostimulatory $\mathrm{CpG}$ oligonucleotides," ACS Applied Materials \& Interfaces, vol. 6, no. 10, pp. 7926-7932, 2014.

[4] C. Bussy, H. Ali-Boucetta, and K. Kostarelos, "Safety considerations for graphene: lessons learnt from carbon nanotubes," Accounts of Chemical Research, vol. 46, no. 3, pp. 692-701, 2013.

[5] Y. Li, L. Feng, X. Shi et al., "Surface coating-dependent cytotoxicity and degradation of graphene derivatives: towards the design of non-toxic, degradable nano-graphene," Small, vol. 10, no. 8, pp. 1544-1554, 2014.

[6] B. Wan, Z.-X. Wang, Q.-Y. Lv et al., "Single-walled carbon nanotubes and graphene oxides induce autophagosome accumulation and lysosome impairment in primarily cultured murine peritoneal macrophages," Toxicology Letters, vol. 221, no. 2, pp. 118-127, 2013.

[7] C. Y. Lee, Q. V. Le, C. Kim, and S. Y. Kim, "Use of silanefunctionalized graphene oxide in organic photovoltaic cells and organic light-emitting diodes," Physical Chemistry Chemical Physics, vol. 17, no. 14, pp. 9369-9374, 2015.

[8] H. Wang, Q. Hao, X. Yang, L. Lu, and X. Wang, "Effect of graphene oxide on the properties of its composite with polyaniline," ACS Applied Materials and Interfaces, vol. 2, no. 3, pp. 821$828,2010$.
[9] T. Kuila, P. Khanra, A. K. Mishra, N. H. Kim, and J. H. Lee, "Functionalized-graphene/ethylene vinyl acetate co-polymer composites for improved mechanical and thermal properties," Polymer Testing, vol. 31, no. 2, pp. 282-289, 2012.

[10] G. Wang, X. Shen, B. Wang, J. Yao, and J. Park, "Synthesis and characterisation of hydrophilic and organophilic graphene nanosheets," Carbon, vol. 47, no. 5, pp. 1359-1364, 2009.

[11] J. H. Shin, S. G. Han, J. K. Kim et al., "5-Day repeated inhalation and 28-day post-exposure study of graphene," Nanotoxicology, pp. 1-9, 2015.

[12] M. Zhang, M. Yang, C. Bussy, S. Iijima, K. Kostarelos, and M. Yudasaka, "Biodegradation of carbon nanohorns in macrophage cells," Nanoscale, vol. 7, no. 7, pp. 2834-2840, 2015.

[13] N. Luo, D. Ni, H. Yue, W. Wei, and G. Ma, "Surfaceengineered graphene navigate divergent biological outcomes toward macrophages," ACS Applied Materials \& Interfaces, vol. 7, no. 9, pp. 5239-5247, 2015.

[14] J. Linares, M. C. Matesanz, M. Vila et al., "Endocytic mechanisms of graphene oxide nanosheets in osteoblasts, hepatocytes and macrophages," ACS Applied Materials \& Interfaces, vol. 6, no. 16, pp. 13697-13706, 2014.

[15] V. Volarevic, V. Paunovic, Z. Markovic et al., "Large graphene quantum dots alleviate immune-mediated liver damage," ACS Nano, vol. 8, no. 12, pp. 12098-12109, 2014.

[16] Y. Qin, Z.-W. Zhou, S.-T. Pan et al., "Graphene quantum dots induce apoptosis, autophagy, and inflammatory response via p38 mitogen-activated protein kinase and nuclear factor- $\kappa \mathrm{B}$ mediated signaling pathways in activated THP-1 macrophages," Toxicology, vol. 327, pp. 62-76, 2015.

[17] M. J. Feito, M. Vila, M. C. Matesanz et al., "In vitro evaluation of graphene oxide nanosheets on immune function," Journal of Colloid and Interface Science, vol. 432, pp. 221-228, 2014.

[18] D. Glick, S. Barth, and K. F. Macleod, "Autophagy: cellular and molecular mechanisms," The Journal of Pathology, vol. 221, no. 1, pp. 3-12, 2010.

[19] A. Haimovici, D. Brigger, B. E. Torbett, M. F. Fey, and M. P. Tschan, "Induction of the autophagy-associated gene MAP1S via PU.1 supports APL differentiation," Leukemia Research, vol. 38, no. 9, pp. 1041-1047, 2014.

[20] D. Brigger, T. Proikas-Cezanne, and M. P. Tschan, "WIPIDependent autophagy during neutrophil differentiation of NB4 acute promyelocytic leukemia cells," Cell Death \& Disease, vol. 5, no. 7, Article ID e1315, 2014.

[21] S. Ueno, H. Tatetsu, H. Hata et al., "PU.1 induces apoptosis in myeloma cells through direct transactivation of TRAIL," Oncogene, vol. 28, no. 46, pp. 4116-4125, 2009.

[22] M. Zhao, X.-F. Duan, D.-H. Wen, and G.-Q. Chen, "PU.1, a novel capase-3 substrate, partially contributes to chemotherapeutic agents-induced apoptosis in leukemic cells," Biochemical and Biophysical Research Communications, vol. 382, no. 3, pp. 508513, 2009. 

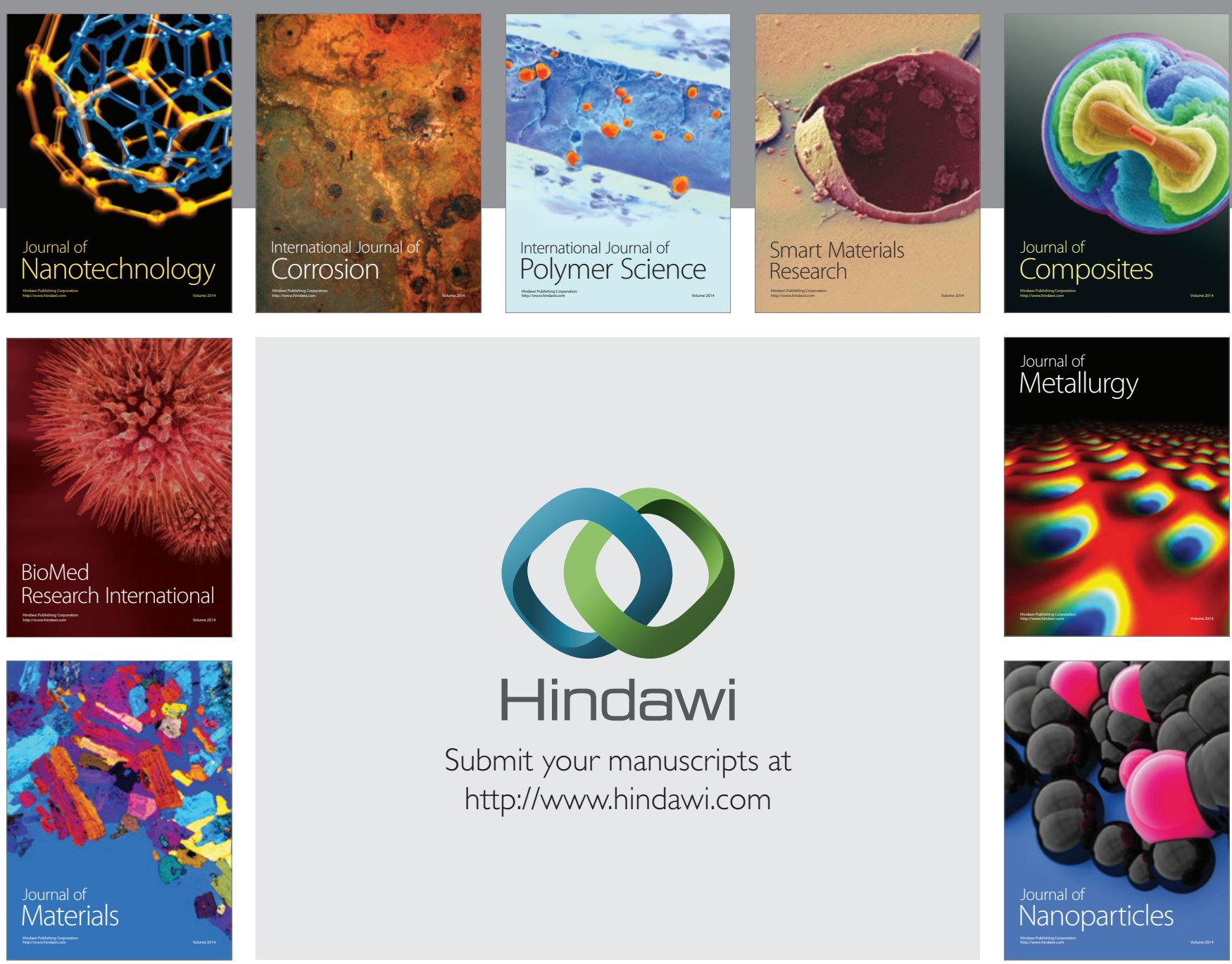

Submit your manuscripts at http://www.hindawi.com
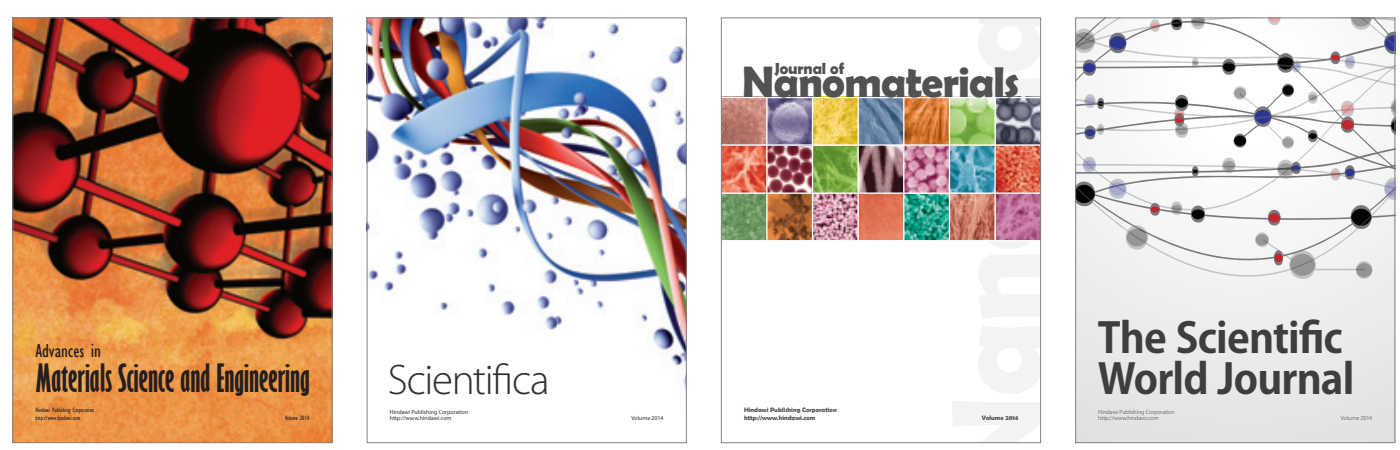

\section{The Scientific World Journal}
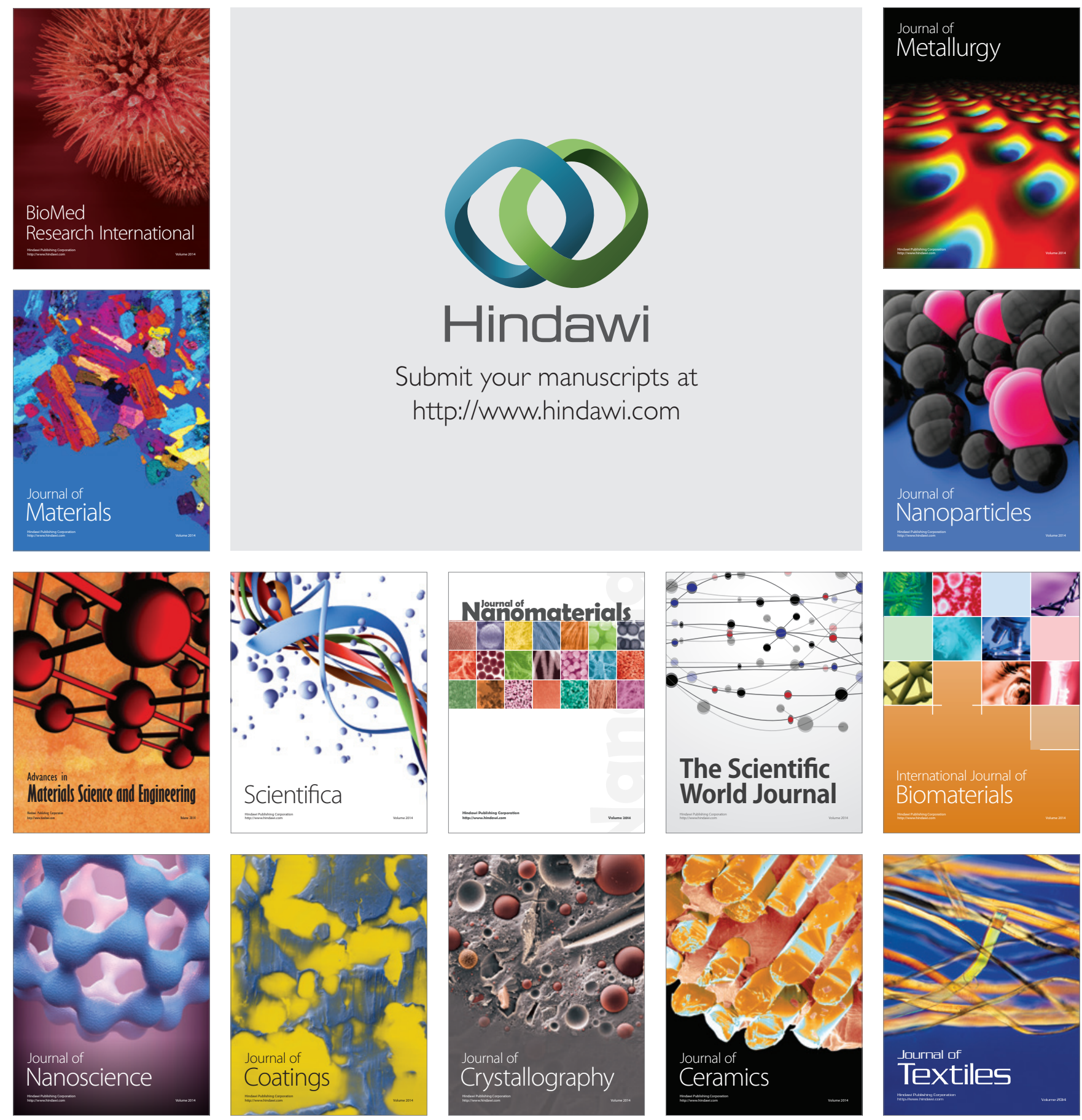